\title{
A bkürzungen.
}

ADHGB. = Allgemeines Deutsches Handelsgesetzbuch.

ALR. = Allgemeines Landrecht für die PreuBischen Staaten.

AN. = Amtliche Nachrichten des Reichs-Versicherungsamtes.

BGB. = Bürgerliches Gesetzbuch.

BUVG. = Bau-Unfallversicherungsgesetz.

CPO. = CivilprozeBordnung.

EEE. = EaER s Eisenbahnrechtliche Entscheidungen.

EG. = Einführungsgesetz.

GVG. = Gerichtsverfassungsgesetz.

GUVG. = Gewerbe-Unfallversicherungsgesetz.

HGB. $=$ Handelsgesetzbuch.

HPG. = Haftpflichtgesetz.

KO. = Konkursordnung.

KVG. = Krankenversicherungsgesetz.

LVA. = Landes-Versicherungsamt.

LUVG. = Unfallversicherungsgesetz für Land- und Forstwirtschaft.

RE. = Rekursentscheidung des Reichs-Versicherungsamtes.

RE. ohne
besonderen
Zusatz $\left\{\begin{array}{l}\text { Vgl. Sammlung der Entscheidungen des } \\ \text { Reichs-Versicherungsamtes oder der Landes- } \\ \text { Versicherungsämter in Unfallentschädigungs- } \\ \text { angelegenheiten, Anlage zum „KompaB“, } \\ \text { Organ der Knappschafts-Berufsgenossen- } \\ \text { schaft für das Deutsche Reich. }\end{array}\right.$

RE. = Reichsgerichtsentscheidung.

RGBl. = Reichsgesetzblatt.

ROHG. = Entscheidung des Reichsoberhandelsgerichtes.

RTDrS. = Reichstagsdrucksachen.

RVA. = Reichs-Versicherungsamt.

StGB. = Strafgesetzbuch.

StPO. = StrafprozeBordnung.

SUVG. = See-Unfallversicherungsgesetz. 